04

\title{
Определение показателей преломления слоистой среды при импульсном режиме облучения
}

\author{
(С) И.П. Яровенко ${ }^{1,2}$, И.В. Прохоров ${ }^{1,2, \text { ฯ }}$ \\ ${ }^{1}$ Институт прикладной математики Дальневосточного отделения РАН, \\ 690041 Владивосток, Россия \\ ${ }^{2}$ Дальневосточный федеральный университет, \\ 690950 Владивосток, Россия \\ I e-mail: prokhorov_iv@mail.ru
}

Поступила в редакцию 31.10.2017 г.

Рассмотрены вопросы определения показателей преломления слоистых сильно-рассеивающих биологических сред методами оптической томографии. В рамках нестационарной модели переноса излучения предложены методы реконструкции показателей преломления слоев, основанные на свойствах гладкости решения начально-краевой задачи. Проведен численный анализ влияния длительности зондируемого импульса на точность восстановления искомых характеристик среды.

DOI: $10.21883 /$ OS.2018.04.45756.254-17

\section{Введение}

Фотонное излучение, проходя через биологическую ткань, взаимодействует с веществом и содержит в себе информацию о структуре объекта и его оптических характеристиках [1]. Восстановить всю информацию об объекте без дополнительных ограничений вряд ли представляется возможным как с практической, так и с теоретической точек зрения. Теоретически это обусловлено множеством известных примеров неединственности решений обратных задач для уравнений, описывающих процессы взаимодействия излучения с веществом [2-4]. На практике ситуация может усугубляться несовершенством измерительной аппаратуры и неполнотой исходных данных. В связи с этим особенно ценны методы томографии, позволяющие найти хотя бы часть данных об исследуемом объекте при минимуме информации о других неизвестных характеристиках среды.

В последние годы широкое развитие получили методы, основанные на использовании генерации ультракоротких импульсов и модуляции с большой частотой лазерного излучения. Использование методов импульсномодуляционной и когерентной оптической томографии позволяет решать задачи раннего обнаружения раковых опухолей и других патологий биологических тканей [5].

В настоящей работе рассмотрена возможность применения импульсного излучения для нахождения показателей преломления слоистой среды по данным об отраженном потоке излучения. Остальные характеристики материалов, такие как коэффициенты ослабления и рассеяния слоев, неизвестны в данной постановке задачи, но и не подлежат определению. Исследование проводится в рамках нестационарного уравнения переноса излучения. Нестационарное уравнение переноса излучения и его приближения являются адекватными моделями для описания транспорта фотонов в рассеивающих и по- глощающих средах [6-8]. Часто уравнение дополняется френелевскими условиями сопряжения на границе раздела сред, полностью определяемыми показателями преломления смежных слоев [9]. Подобные модели широко используются не только в томографии биологических тканей, но и в атмосферной оптике, высокочастотной акустике флуктуирующих сред и других приложениях теории переноса излучения [1,9-11]. Вопросы корректности начально-краевых задач для стационарных и нестационарных уравнений переноса излучения с условиями отражения и преломления на границах раздела сред исследованы в работах [12-19].

В рамках этой модели авторами исследованы обратные и экстремальные задачи для стационарного уравнения переноса неполяризованного излучения [20-23]. Содержание обратных задач заключалось в нахождения показателей преломления слоистой среды, а постановки экстремальных задач имели цель определения оптимальных параметров иммерсионной жидкости при оптическом просветлении биологических тканей. В работе [24] результаты исследования обратных задач для скалярного уравнения обобщены на векторный случай, описывающий процесс зондирования биоткани поляризованным потоком излучения. Упомянутые выше работы объединяет общий подход к исследованию задач оптической томографии. Он основан на исследовании качественных свойств решений уравнения переноса излучения и выделении характерных особенностей решения, обусловленных явлением полного внутреннего отражения на границе раздела сред. В работе предложен метод обработки выходящего сигнала, состоящий в построении некоторой скалярной индикаторной функции, имеющей неограниченный рост вблизи искомых показателей преломления слоев. Рассмотрение нестационарного процесса зондирования позволяет увеличить эффективность предлагаемого метода, варьируя длительность импульса 
источника излучения. На ряде численных экспериментов продемонстрированы влияние длительности зондирующего сигнала на качество восстановления показателей преломления.

\section{Постановка задачи}

Рассматривается процесс распространения монохроматического излучения в бесконечной по направлениям $x$ и $y$ среде, имеющей плоскопараллельное строение. Среда состоит из $n$ пластин $G_{i}=\left(z_{i-1}, z_{i}\right), i=1, \ldots n$, обладающих различными оптическими характеристиками, и пусть $G_{0}=\bigcup_{i=1}^{n} G_{i}$. С учетом симметрии плотность потока излучения будет зависеть только от двух переменных: пространственной $z$ и угловой $v$, где $v-$ косинус угла между положительным направлением оси $z$ и направлением распространения фотона. Нестационарный процесс излучения в такой среде может быть описан с помощью следующего уравнения [21]:

$$
\begin{aligned}
\left(\frac{1}{v(z)} \frac{\partial}{\partial t}\right. & \left.+v \frac{\partial}{\partial z}+\mu(z)\right) f(z, v, t) \\
& =\mu_{s}(z) \int_{-1}^{1} p\left(z, v, v^{\prime}\right) f\left(z, v^{\prime}, t\right) d v^{\prime}
\end{aligned}
$$

Функция $f(z, v, t)$ интерпретируется как плотность потока фотонов в момент времени $t \in\left[0, t_{\max }\right]$, в точке $z \in G=\left(z_{0}, z_{n}\right)$, движущихся со скоростью $v$ в направлении $v \in[-1,1]$. Функции $\mu$ и $\mu_{s}$ имеют смысл коэффициентов ослабления и рассеяния соответственно, а $v(z)$ характеризует скорость распространения излучения в веществе. Внутри каждого слоя $G_{i}$ коэффициенты уравнения постоянны: $v(z)=v_{i}, \mu(z)=\mu_{i}, \mu_{s}(z)=\mu_{s_{i}}$.

Величина $p\left(z, v, v^{\prime}\right)$ называется фазовой функцией и определяет характер рассеяния фотонов на частицах среды. При моделировании единичных актов светорассеяния в биологической среде зачастую используют функцию Хеньи-Гринстейна [1]:

$$
\begin{gathered}
p(\theta)=\frac{1-\bar{g}}{\left(1+\bar{g}^{2}-2 \bar{g} \cos \theta\right)^{3 / 2}}, \\
\cos \theta=v v^{\prime}+\sqrt{\left(1-v^{2}\right)\left(1-v^{\prime 2}\right)}
\end{gathered}
$$

где величина $\bar{g} \in[-1,1]$ характеризует степень анизотропии рассеяния в среде. Случай $\bar{g}=0$ соответствует изотропному рассеянию, $\bar{g}=1-$ полному рассеянию вперед, $\bar{g}=-1$ - полному рассеянию назад.

Уравнение (1) дополняется граничными и начальными условиями

$$
\begin{gathered}
f\left(z_{0}, v, t\right)=h(v, t), v \in(0,1], \\
f\left(z_{n}, v, t\right)=0, v \in[-1,0),
\end{gathered}
$$

$$
f(z, v, 0)=0, \quad z \in G_{0}, v \in[-1,1] .
$$

Функция $h$ в условии (2) описывает плотность потока входящего в среду излучения через поверхность $z=z_{0}$, а соотношение (3) означает, что в начальный момент времени источники излучения в среде отсутствовали. В дальнейшем будем полагать, что функция $h$ имеет ограниченные производные по переменной $v$.

На границах раздела сред $z=z_{i}, i=1, \ldots, n-1$ ставятся условия сопряжения, являющиеся следствием формул Френеля $[8,11,14,17]$ :

$$
\begin{aligned}
f\left(z_{i}+0, v, t\right)= & R_{i}(v) f\left(z_{i}+0,-v, t\right) \\
& +T_{i}(v) f\left(z_{i}-0, \psi_{i}, t\right), v \in[-1,0), \\
f\left(z_{i}-0, v, t\right)= & R_{i}(v) f\left(z_{i}-0,-v, t\right) \\
& +T_{i}(v) f\left(z_{i}+0, \psi_{i}, t\right), v \in(0,1],
\end{aligned}
$$

где $f(z \pm 0, v, t)=\lim _{\varepsilon \rightarrow 0, \varepsilon>0} f(z \pm \varepsilon, v, t)$ - соответствующие предельные значения функции $f(z, v, t)$ при стремлении точки $z$ к границе слоя.

Коэффициенты $R_{i}(v), T_{i}(v)$ характеризуют отражательную и пропускную способность поверхности раздела $z=z_{i}, i=1, \ldots, n-1$, и для неполяризованного излучения определяются следующими формулами:

$$
\begin{gathered}
R_{i}(v)=\frac{1}{2}\left(R_{\|, i}^{2}+R_{\perp, i}^{2}\right), \quad T_{i}(v)=1-R_{i}(v), \\
R_{\|, i}(v)=\frac{\widetilde{\kappa}_{i}(v) \psi_{i}(v)-v}{\widetilde{\kappa}_{i}(v) \psi_{i}(v)+v}, \quad R_{\perp, i}(v)=\frac{\psi_{i}(v)-\widetilde{\kappa}_{i}(v) v}{\psi_{i}(v)+\widetilde{\kappa}_{i}(v) v},
\end{gathered}
$$

где $\widetilde{\kappa}_{i}(v)$ - относительный коэффициент преломления, определяемый через показатели преломления $\kappa_{i}$ и $\kappa_{i+1}$ слоев $G_{i}$ и $G_{i+1}$ :

$$
\widetilde{\kappa}_{i}(v)= \begin{cases}\kappa_{i+1} / \kappa_{i}, & \text { при } 0<v \leq 1, \\ \kappa_{i} / \kappa_{i+1}, & \text { при }-1 \leq v<0,\end{cases}
$$

а величина $\psi_{i}=\psi_{i}(v)$ связана с $v$ законом Снеллиуса:

$$
\psi_{i}(v)=\left\{\begin{aligned}
& \operatorname{sign}(v) \sqrt{1-\widetilde{\kappa}_{i}^{2}(v)\left(1-v^{2}\right),} \\
& \text { при } 1-\widetilde{\kappa}_{i}^{2}(v)\left(1-v^{2}\right) \geq 0, \\
& 0, \quad \text { при } 1-\widetilde{\kappa}_{i}^{2}(v)\left(1-v^{2}\right)<0 .
\end{aligned}\right.
$$

Из определения величины $\psi_{i}(v)$ видно, что для всех $v$ таких, что выполняется неравенство $1-\widetilde{\kappa}_{i}^{2}(v)\left(1-v^{2}\right) \leq 0$, справедливы соотношения $T_{i}(v)=0, R_{i}(v)=1$. В оптике данный случай носит название полного внутреннего отражения [9].

Рассмотрим следующую задачу. Определить абсолютные показатели преломления $\kappa_{i}$ слоистой среды из уравнения (1), граничных условий (2), начального условия (3), условий сопряжения (4), и дополнительного 
условия на границе

$$
\int_{0}^{t_{\max }} f\left(z_{0}-0, v, t\right) d t=H\left(z_{0}, v\right), \quad v \in[-1,0),
$$

если известен показатель преломления первого слоя $\kappa_{1}$ и функция $H$.

Таким образом, задача заключается в нахождении абсолютных показателей преломления материалов входящих в состав многокомпонентной среды в случае, когда известно лишь осредненное по времени выходящее излучение.

\section{Метод нахождения показателей преломления}

В данном разделе излагается способ определения показателей преломления слоев, входящих в состав многокомпонентной среды. Идея метода определения показателей преломления основана на использовании особенности решения уравнения переноса излучения по угловой переменной вблизи угла полного внутреннего отражения. Как известно, при приближении угла $\theta=\arccos (v)$ к углу полного внутреннего отражения $\theta_{0}=\arccos \left(v_{0}\right)$, коэффициенты отражения и пропускания меняются очень быстро, но остаются непрерывными [21].

Дифференцируя соотношение (5) при $\widetilde{\kappa}>1$, получаем

$$
\begin{aligned}
R_{i}^{\prime}(v) & =R_{\|, i}^{\prime} R_{\|, i}+R_{\perp, i}^{\prime} R_{\perp, i} \\
& =2 \frac{\widetilde{\kappa}_{i}^{3}-\widetilde{\kappa}_{i}}{\psi_{i}(v)}\left(\frac{R_{\|, i}}{\left(\widetilde{\kappa}_{i} \psi_{i}(v)+v\right)^{2}}+\frac{R_{\perp, i}}{\left(\psi_{i}(v)+\widetilde{\kappa}_{i} v\right)^{2}}\right) .
\end{aligned}
$$

Очевидно, что знаменатели выражения в скобках не могут обращаться в нуль в силу того, что знаки величин $v$ и $\psi_{i}(v)$ совпадают и выполняется условие $v<v_{0}\left(\widetilde{\kappa}_{i}\right)$. В то же время, $\psi_{i}(v) \rightarrow-\infty$ при $v \rightarrow v_{0}\left(\widetilde{\kappa}_{i}-0\right)$. Отсюда, учитывая ограниченность коэффициентов $R_{\|, i}, R_{\perp, i}$ и справедливость соотношения $R^{\prime}+T^{\prime}=0$, получаем, что $R_{i}^{\prime}(v) \rightarrow-\infty$ при $v \rightarrow v_{0}\left(\widetilde{\kappa}_{i}-0\right), T_{i}^{\prime}(v) \rightarrow \infty$ при $\nu \rightarrow v_{0}\left(\widetilde{\kappa}_{i}-0\right)$.

Выясним как влияет неограниченность производных коэффициентов отражения и пропускания на поведение производной выходящего излучения. Для этого проинтегрируем уравнение (1) и условия $(2),(4)$ по переменной $t \in\left[0, t_{\max }\right]$, получаем

$$
\begin{aligned}
& \frac{1}{v}\left(f\left(z, v, t_{\max }\right)-f(z, v, 0)\right)+\left(v \frac{\partial}{\partial z}+\mu(z)\right) \\
& \times \int_{0}^{t_{\max }} f(z, v, t) d t=\mu_{s}(z) \int_{-1}^{1} p\left(z, v, v^{\prime}\right) \int_{0}^{t_{\max }} f\left(z, v^{\prime}, t\right) d t d v^{\prime} .
\end{aligned}
$$

В силу того, что источник излучения предполагается импульсным, величина $f\left(z, v, t_{\max }\right)$ будет убывать с ростом $t_{\max }$. Пусть $t_{\max }$ настолько велико, что можно пренебречь величиной $f\left(z, v, t_{\max }\right)$ в уравнении (8). Тогда, учитывая начальное условие (3) и вводя обозначение

$$
f_{0}(z, v)=\int_{0}^{t_{\max }} f(z, v, t) d t
$$

придем к краевой задаче относительно функции $f_{0}$, которая по своей форме аналогична краевой задаче для стационарного уравнения переноса излучения. Функция $f_{0}$ удовлетворяет уравнению

$$
\begin{aligned}
v \frac{\partial}{\partial z} f_{0}(z, v) & +\mu(z) f_{0}(z, v) \\
& =\mu_{s}(z) \int_{-1}^{1} p\left(z, v, v^{\prime}\right) f_{0}\left(z, v^{\prime}\right) d v^{\prime}
\end{aligned}
$$

условиям на внешней границе неоднородного слоя

$$
f_{0}\left(z_{0}, v\right)=\int_{0}^{t_{\max }} h(v, t) d t, \quad f_{0}\left(z_{n}, v\right)=0
$$

и условиям сопряжения на границе раздела сред $z=z_{i}, i=1, \ldots, n-1$

$$
\begin{aligned}
f_{0}\left(z_{i}+0, v\right)= & R_{i}(v) f_{0}\left(z_{i}+0,-v\right) \\
& +T_{i}(v) f_{0}\left(z_{i}-0, \psi_{i}\right), v \in[-1,0), \\
f_{0}\left(z_{i}-0, v\right)= & R_{i}(v) f_{0}\left(z_{i}-0,-v\right) \\
& +T_{i}(v) f_{0}\left(z_{i}+0, \psi_{i}\right), v \in(0,1] .
\end{aligned}
$$

Для краевой задачи (9)-(11) в произвольной точке слоя $G_{i}=\left(z_{i-1}, z_{i}\right)$ справедливо следующее представление [21,22]:

$$
\begin{aligned}
& f_{0}(z, v)=\left[R_{i-1}(v) f_{0}\left(z_{i-1}-0,-v\right)\right. \\
& \left.+T_{i-1}(v) f_{0}\left(z_{i-1}+0, \psi_{i-1}(v)\right)\right] e^{-\frac{\mu_{i}}{|v|}\left(z-z_{i-1}\right)} \\
& +\frac{1}{v} \int_{z_{i-1}}^{z} e^{-\frac{\mu_{i}}{|v|}\left(z^{\prime}-z_{i-1}\right)} \mu_{s, i} \\
& \times \int_{-1}^{1} p\left(z^{\prime}, v, v^{\prime}\right) f_{0}\left(z^{\prime}, v^{\prime}\right) d v^{\prime} d z^{\prime}, v<0, \\
& f_{0}(z, v)=\left[R_{i}(v) f_{0}\left(z_{i}+0,-v\right)+T_{i}(v)\right. \\
& \left.\times f_{0}\left(z_{i}-0, \psi_{i}(v)\right)\right] e^{-\frac{\mu_{i}}{|v|}\left(z_{i}-z\right)}+\frac{1}{v} \int_{z_{i}}^{z} e^{-\frac{\mu_{i}}{|v|}\left(z_{i}-z^{\prime}\right)} \\
& \times \mu_{s, i} \int_{-1}^{1} p\left(z^{\prime}, v, v^{\prime}\right) f_{0}\left(z^{\prime}, v^{\prime}\right) d v^{\prime} d z^{\prime}, v>0 .
\end{aligned}
$$


Дифференцируя $f_{0}(z, v)$ по угловой переменной $v$, из (12) можно получить следующие выражения для производных от выходящих из слоя потоков излучения на нижней и верхней границах соответственно:

$$
\begin{aligned}
& \frac{\partial}{\partial v} f_{0}\left(z_{i-1}+0, v\right)=K_{i}(v)\left[\frac{\partial}{\partial v} R_{i-1}(v) M_{i-1}(v)\right. \\
& -\frac{\partial}{\partial v} R_{i}(-v) R_{i-1}(v) M_{i}(-v) e^{-\tau_{i, i-1}}-R_{i-1}(v) S_{i}(-v) \\
& \times \frac{\partial}{\partial v} f_{0}\left(z_{i}+0, \psi_{i}(-v)\right) e^{-\tau_{i, i-1}}+S_{i-1}(v) \\
& \left.\times \frac{\partial}{\partial v} f_{0}\left(z_{i-1}-0, \psi_{i-1}(v)\right)\right] e^{-\tau_{i, i-1}}+O(1), \quad v<0 \\
& \frac{\partial}{\partial v} f_{0}\left(z_{i}-0, v\right)=K_{i}(-v)\left[\frac{\partial}{\partial v} R_{i}(v) M_{i}(v)\right. \\
& -\frac{\partial}{\partial v} R_{i-1}(-v) R_{i}(v) M_{i-1}(-v) e^{-\tau_{i, i-1}}-R_{i}(v) S_{i-1}(-v) \\
& \times \frac{\partial}{\partial v} f_{0}\left(z_{i-1}-0, \psi_{i-1}(-v)\right) e^{-\tau_{i, i-1}} \\
& \left.+S_{i}(v) \frac{\partial}{\partial v} f_{0}\left(z_{i}+0, \psi_{i}(v)\right)\right] e^{-\tau_{i, i-1}}+O(1), v>0
\end{aligned}
$$

В (13),(14) величина $O(1)$ обозначает ограниченную функцию относительно переменной $v$, а через $M_{i}, S_{i}, K_{i}, \tau_{i, i-1}$ обозначены следующие величины:

$$
\begin{gathered}
M_{i}(v)=f_{0}\left(z_{i},-v\right)-f_{0}\left(z_{i}, \psi_{i}(v)\right), \\
S_{i}(v)=T_{i}(v) \frac{\partial \psi_{i}}{\partial v}, \\
K_{i}(v)=\frac{1}{1-R_{i-1}(v) R_{i}(-v) \exp -2 \tau_{i, i-1}}, \\
\tau_{i, i-1}=\frac{\mu_{i}}{|v|}\left(z_{i}-z_{i-1}\right) .
\end{gathered}
$$

Из представлений (7),(13), (14) вытекает, что внутри слоя $G_{i}$ у производной функции $f_{0}$ могут возникать особенности из-за неограниченности производных коэффициентов отражения $R_{i-1}(v)$ и $R_{i}(v)$. В результате переотражений в слой могут «приходить» особенности из соседних слоев $G_{i-1}$ и $G_{i+1}$ за счет величин

$$
\frac{\partial}{\partial v} f_{0}\left(z_{i} \pm 0, \psi_{i}(v)\right), \quad \frac{\partial}{\partial v} f_{0}\left(z_{i-1} \pm 0, \psi_{i-1}(v)\right),
$$

поскольку для них справедливы аналогичные формулы. Заметим, что косинус угла, при котором возникает особенность из-за полного внутреннего отражения на границе $z=z_{i}$, будет изменяться при переходе через границы $z=z_{j}, 0<j<i$ согласно закону Снеллиуса. Поскольку особенность в коэффициенте $R_{i}^{\prime}$ определяется величиной вида $1 / \psi_{i}(v)$, то для $R_{i}^{\prime}\left(\psi_{i-1}\right)$ особенность будет обусловлена выражением $1 / \psi_{i}\left(\psi_{i-1}(v)\right)$. Нетрудно увидеть, что

$$
\psi_{i}\left(\psi_{i}-1\left(\ldots \psi_{1}(v)\right)\right)=\operatorname{sign}(v) \sqrt{1-\left(\kappa_{1} / \kappa_{i}\right)^{2}\left(1-v^{2}\right)} .
$$

Поэтому особенность у прошедшего потока будет иметь место при приближении аргумента $v$ к косинусу угла полного внутреннего отражения, определяемого отношением $\kappa_{1} / \kappa_{i}$. Отметим следующее важное обстоятельство. Особенность от некоторого слоя с номером $i, 2 \leq i \leq n$ „дойдет“ до границы $z=z_{0}$ лишь в том случае, если ни одна из функций $\psi_{j}, j=1, \ldots, i$ в комбинации (15) не обращается в ноль. Из этого требования вытекают ограничения на показатели преломления слоистой среды, при которых возможно определение показателя преломления $i$-го слоя:

$$
\frac{k_{i}}{k_{1}}<1, \quad \frac{k_{i}}{k_{j}} \leq 1, j=2, \ldots, i, \frac{k_{i}}{k_{i+1}}<1 .
$$

В (16) последнее неравенство является следствием условия возникновения полного внутреннего отражения на границе $z=z_{i}$, а остальные условия обеспечивают неравенство нулю коэффициентов перед производной соответствующего коэффициента отражения в (13), (14).

Зная косинусы углов, при которых происходит полное внутреннее отражение, нетрудно определить показатели преломления, соответствующие этим углам. Подобно тому, как предлагалось в $[21,22]$, для нахождения показателей преломления можно следовать следующему алгоритму.

1. Вычислить производную по переменной $v$ от функции $f_{0}\left(z_{0}, v\right)=H\left(z_{0}, v\right), v<0$.

2. Найти точки $v_{0}^{(1)}, v_{0}^{(2)}, \ldots, v_{0}^{(n-1)}$, где производная принимает аномально большие значения.

3. Определить искомые показатели преломления с помощью формул

$$
\kappa_{i}=\kappa_{1} \sqrt{1-\left(v_{0}^{(i)}\right)^{2}}, i=2, \ldots, n-1 .
$$

Ниже рассмотрим другой способ определения показателей преломления, который на наш взгляд имеет ряд преимуществ. Для этого определим функцию

$$
\begin{gathered}
\operatorname{Ind}(\kappa)=\int_{-1}^{0}\left|\alpha(v, \kappa) \frac{\partial H}{\partial v}\left(z_{0}, v\right)\right| d v, \\
\alpha(\nu, \kappa)= \begin{cases}\frac{v}{\sqrt{1-\left(\frac{\kappa_{1}}{\kappa}\right)^{2}\left(1-v^{2}\right)},} & v<-\sqrt{1-\left(\frac{\kappa}{\kappa_{1}}\right)^{2}}, \\
0, & v>-\sqrt{1-\left(\frac{\kappa}{\kappa_{1}}\right)^{2}} .\end{cases}
\end{gathered}
$$

Подставляя представление (13), (14) в формулу (18) нетрудно показать, что при выполнении условий (16) 
Оптические характеристики многослойной среды

\begin{tabular}{l|c|c|c|c|c}
\hline \multicolumn{1}{c|}{ Слой } & $\begin{array}{c}\text { Толщина, } \\
\mu \mathrm{m}\end{array}$ & $\begin{array}{c}\text { Коэффициент } \\
\text { ослабления, } \mathrm{cm}^{-1}\end{array}$ & $\begin{array}{c}\text { Коэффициент } \\
\text { рассеяния, } \mathrm{cm}^{-1}\end{array}$ & $\begin{array}{c}\text { Показатель } \\
\text { преломления }\end{array}$ & $\begin{array}{c}\text { Фактор } \\
\text { анизотропии }\end{array}$ \\
\hline Эпидермис & 65 & 11.13 & 10.7 & 1.5 & 0.8 \\
Верхняя дерма & 565 & 18.97 & 18.7 & 1.4 & 0.72 \\
Капиллярные сплетения & 90 & 42.5 & 40.0 & 1.35 & 0.72
\end{tabular}

вблизи точки $\kappa=\kappa_{i}$ справедливо следующее разложение:

$$
\operatorname{Ind}(k)=A \ln \left|\frac{\kappa_{i}-\kappa}{\kappa_{i}+\kappa}\right|+O(1),
$$

где $A-$ некоторая ограниченная функция. Из данного разложения видно, что функция $\operatorname{Ind}(\kappa)$ будет неограниченно возрастать при приближении аргумента $\kappa$ к искомому значению показателя преломления $\kappa_{i}$.

Ядро интегрального преобразования (18) устроено таким образом, что оно переводит особенность по угловой переменной в выходящем излучении в особенность у функции $\operatorname{Ind}(\kappa)$ по показателю преломления. C одной стороны, это упрощает обработку сигнала, так как не нужно вручную отслеживать местоположение особенности у производной и производить пересчет в искомый показатель преломления. С другой стороны, наличие интеграла в формуле (18) приводит к некоторому сглаживанию шумов, содержащихся в исходном сигнале, что тоже положительно сказывается на качестве восстановления показателей преломления.

\section{Численные эксперименты}

Эффективность данного подхода продемонстрирована вычислительным экспериментом определения показателей преломления слоистой среды. Решение задачи включает в себя два этапа. На первом, мы полагаем параметры среды известными, решаем прямую задачу и находим функцию $H$, которая описывает выходящее излучение. Для решения уравнения переноса излучения использовался метод Монте-Карло, описанный в работе [16]. На втором этапе вычисляем функцию $\operatorname{Ind}(\kappa)$ и находим те значения аргумента, при которых функция принимает аномально большие значения. Дифференцирование функции $H$ выполнялось при помощи разностных отношений. Для преодоления хорошо известной неустойчивости численного дифференцирования применялась наиболее простая регуляризация, заключающаяся в согласовании точности вычисления дифференцируемой функции и шага дифференцирования. Эти параметры определялись путем предварительных численных расчетов. Для вычисления интеграла в (18) применялся метод трапеций.

В численных экспериментах рассмотрена следующая трехслойная среда, моделирующая кожный слой: 1) эпидермис, 2) дерма, 3) капиллярные сплетения. Использованы оптические характеристики этой модельной среды, соответствующие длине волны $633 \mathrm{~nm}$ [23]. Параметры среды приведены в таблице.

В качестве источника излучения выбирался гауссиан по времени, имеющий вид

$$
h(z, v, t)=I_{0} \exp \left(-4 \ln 2 \frac{\left(t-t_{0}\right)^{2}}{t_{p}}\right),
$$

где $I_{0}-$ амплитуда импульса, $t_{0}-$ момент времени соответствующий максимальной мощности сигнала. В экспериментах использовались следующие значения указанных величин: $I_{0}=1, t_{0}=0.1$. Величина $t_{p}$ определяет длительность импульса на половине амплитуды и варьируется в экспериментах.

Для выбора значения $t_{\max }$ были построены графики величины

$$
\chi\left(t_{\max }, v\right)=f\left(z_{0}, v, t_{\max }\right) / f_{0}\left(z_{0}, v\right),
$$

характеризующей отклонение плотности потока излучения в конечный момент времени от осредненного по времени потока излучения. График величины $\chi\left(t_{\max }, v\right)$, рассчитанный для длительности сигнала $t_{p}=2 \cdot 10^{-11} \mathrm{~s}$ при значении $v=-1$, соответствующей нормально отраженному потоку, приведен на рис. 1. Как видно из графика, с ростом $t_{\max }$ данная величина достаточно быстро убывает. В результате предварительных расчетов было выбрано значение $t_{\max }=6 \cdot 10^{-11} \mathrm{~s}$, которое использовалось в последующих численных экспериментах.

Результаты экспериментов при облучении исследуемой среды импульсами длительностью

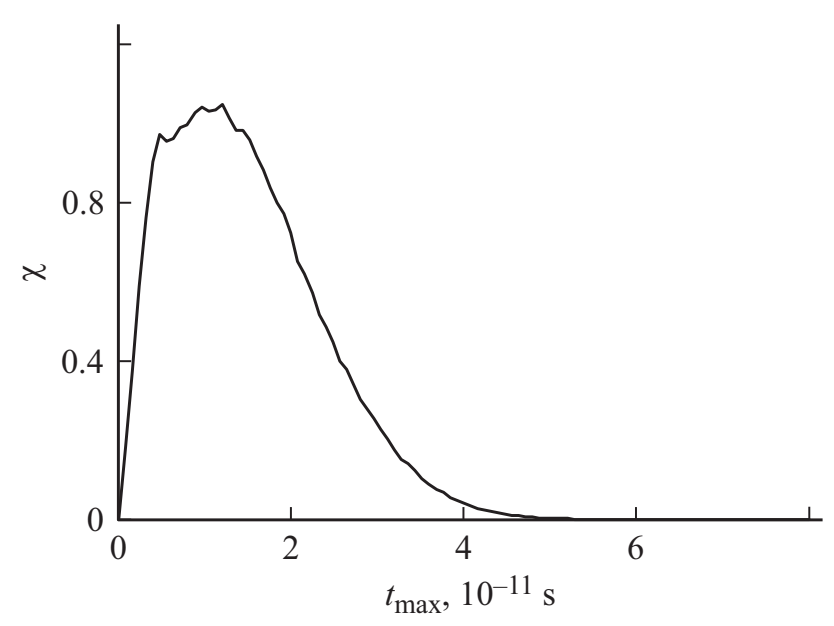

Рис. 1. График величины $\chi\left(t_{\max },-1\right)$ в зависимости от $t_{\max }$ для длительности импульса $2 \cdot 10^{-11} \mathrm{~s}$. 

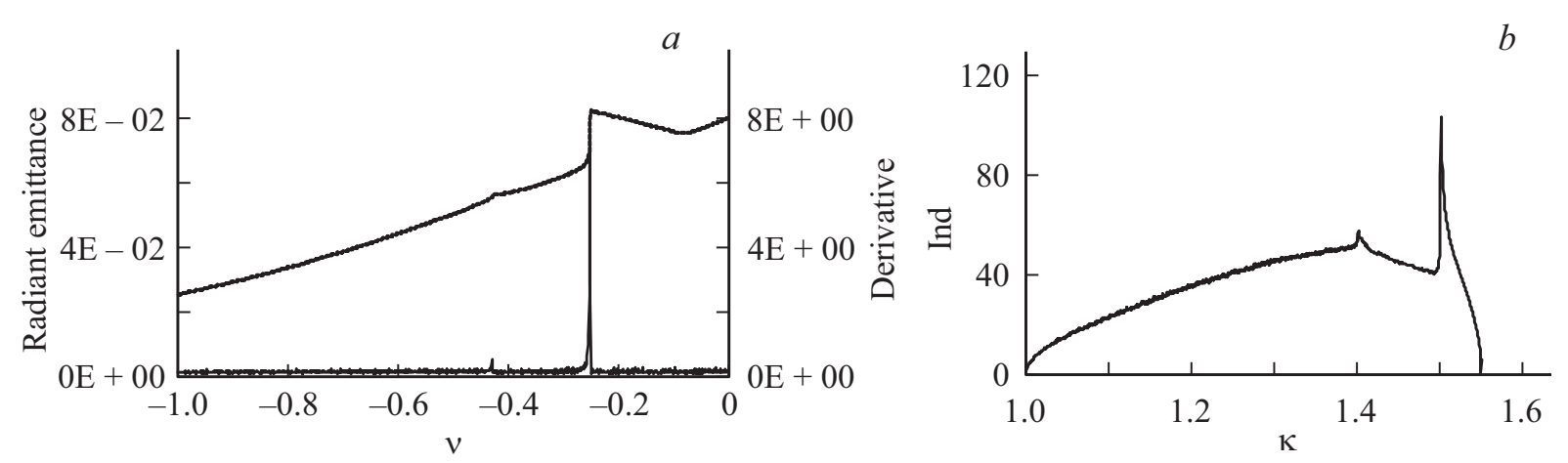

Рис. 2. $a$ - плотность потока выходящего излучения (прерывистая линия) и модуль ее производной (непрерывная линия) в зависимости от угловой переменной $v$ для длительности импульса $5 \cdot 10^{-11} \mathrm{~s}, b-$ функция $\operatorname{Ind}(\kappa)$.
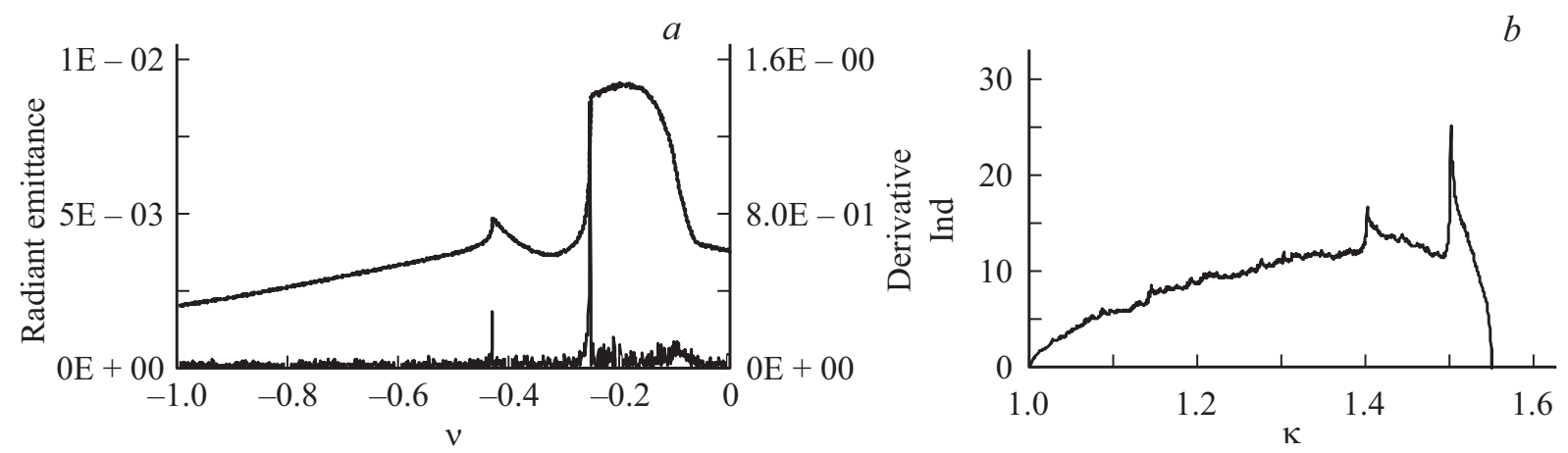

Рис. 3. $a$ - плотность потока выходящего излучения (прерывистая линия) и модуль ее производной (непрерывная линия) в зависимости от угловой переменной $v$ для длительности импульса $2 \cdot 10^{-11} \mathrm{~s}, b-$ функция $\operatorname{Ind}(\kappa)$.
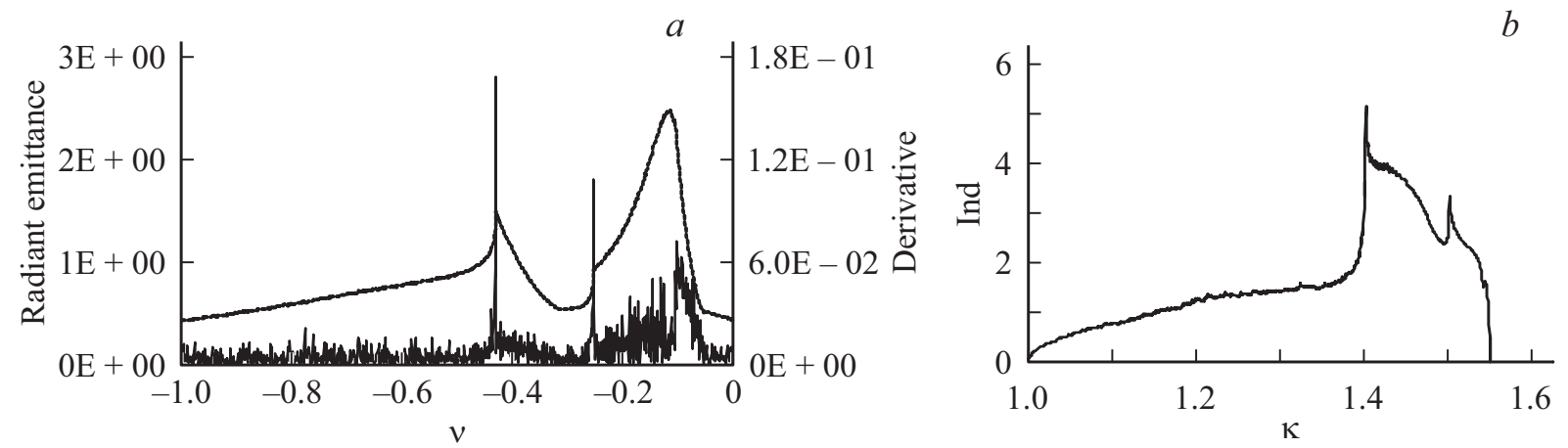

Рис. 4. $a$ - графики плотности потока выходящего излучения (прерывистая линия) и модуля ее производной (непрерывная линия) в зависимости от угловой переменной $v$, для длительности импульса $10^{-11} \mathrm{~s}, b-$ функция $\operatorname{Ind}(\kappa)$.

$t_{p}=(1,2,5) \cdot 10^{-11} \mathrm{~s} \quad$ приведены на рис. $2-4$ соответственно. На рис. 2, $a, 3, a, 4, a$ приведены графики осредненного по времени выходящего излучения (прерывистая линия) и соответствующие им графики производных по угловой переменной (непрерывная линия). Графики $f_{0}$ и ее производной специально представлены на одной картинке, чтобы наглядно продемонстрировать изменения выходящего потока и его производной. На рисунках выходящему потоку соответствуют значения на оси ординат, расположенной слева, а значениям производной - на шкале, размещенной справа. Графики на рисунках под литерой $b$ отражают результат обработки выходящего сигнала с помощью функции Ind.

В силу соотношений (16) особенности, обусловленные полным внутренним отражением в более глубоких слоях, будут соответствовать меньшим значениям угловой переменной. Из рис. $2-4$ видно, что увеличение длительности импульса приводит к тому, что особенность у выходящего излучения, соответствующая более глубокому слою, становится менее выраженной. Так, например, на рис. 2, $a$ пик у производной выходящего потока, соответствующий границе между вторым и третьим слоями, становится едва различим на фоне шума, вызванного 
численным дифференцированием. В то же время метод обработки сигнала с помощью преобразования (18) позволяет восстановить соответствующий ему показатель преломления с хорошим качеством (рис. 2, $b$ ).

Уменьшение длительности импульса, напротив, в целом положительно сказывается на качестве восстановления показателей преломления. Отдельно стоит прокомментировать рисунок, соответствующий ширине импульса $10^{-11} \mathrm{~s}$. Как видно из рис. 4, $a$, отраженный поток достаточно быстро меняется вблизи направления $v=-0.1$, при этом такое изменение не соответствует полному внутреннему отражению ни на одной из границ раздела. Указанное поведение обусловлено тем, что для направлений, близких к касательной, фотонам требуется гораздо больше времени, чтобы „пройти“ через все слои и „выйти“ из среды. Как показывает график на рис. 4, a, численное дифференцирование выходящего сигнала дает пик, соответствующий направлению $v=-0.1$. При непосредственном нахождении показателя преломления по формуле (17) данный пик приведет к появлению ложного значения показателя преломления $\kappa=1.53$, не соответствующего ни одному из слоев исследуемой среды. В тоже время применение индикатора (18) позволяет отфильтровать эту особенность путем специального выбора ядра интегрального преобразования. Ядро преобразования позволяет усиливать особенности, вызванные полным внутренним отражением, а другие особенности, возникающие в выходящем излучении, сглаживаются с помощью операции интегрирования.

\section{Заключение}

Достоинством предложенного в работе метода является возможность определения искомых показателей преломления на основе знания только некоторой интегральной характеристики выходящего из среды излучения без задания других оптических параметров среды. Метод не итерационный, экономичный и не требует выделения баллистической составляющей, что особенно важно при диагностике сильно рассеивающих сред. Стоит сказать несколько слов о длительности импульсов, применяемых для облучения исследуемой среды. В современной лазерной биомедицине сравнительно давно появилась возможность оперировать с фемтосекундными импульсами $[25,26]$. При компьютерном моделировании в настоящей работе рассматривались импульсы длительностью на несколько порядков больше. С одной стороны, это обусловлено выбранной математической моделью, адекватно описывающей процесс взаимодействия некогерентного излучения со средой и справедливой в случае, когда толщина слоистой среды намного больше длины когерентности излучения. С другой стороны, нашей задачей было показать, что даже небольшое влияние нестационарности источника излучения дает заметный выигрыш в качестве восстановления оптических характеристик сред, оставаясь в рамках классической геомет- рической оптики без применения модуляции либо когерентности излучения. Одна из характерных особенностей изучаемой задачи - это большой объем исходных данных, вызванный необходимостью знания выходящего потока излучения на подробной сетке направлений. Это приводит к необходимости применения многоканальной системы сбора и детектирования рассеянного излучения. Регистрация более длительных импульсов упрощает схемотехнику устройств, измеряющих и обрабатывающих отраженный сигнал.

Отдельно стоит прокомментировать условия (16), обеспечивающие единственность решения обратной задачи. Ограничения (16) являются априорной информацией о структуре исследуемой среды и несомненно снижают прикладную ценность предлагаемого метода. Тем не менее в оптике биотканей подобные ситуации не редкость. Зачастую состав среды в целом известен, а требуется определить изменения показателя преломления, вызванные, например, введением лекарственных препаратов или зарождением патологических процессов $[1,27]$.

Работа выполнена при финансовой поддержке Российского научного фонда (проект 14-11-00079).

\section{Список литературы}

[1] Тучин В.В. Оптика биологических тканей. Методы рассеяния света в медицинской диагностике. М.: Физматлит, 2013; Tuchin V.V. Tissue Optics: Light Scattering Methods and Instruments for Medical Diagnosis. Bellingham, Wash. USA: SPIE PRESS, 2007.

[2] Аниконов Д.С., Ковтанюк А.Е., Прохоров И.В. Использование уравнения переноса в томографии. М.: Логос, 2000; Anikonov D.S., Kovtanyuk A.E., Prokhorov I.V. Transport Equation and Tomography. Utrecht-Boston: VSP, 2002.

[3] Аниконов Д.С., Прохоров И.В. // Журнал вычислительной математики и математической физики. 2002. Т. 42. № 3. C. 370-379; Anikonov D.S., Prokhorov I.V. // Computational Mathematics and Mathematical Physics. 2002. V. 42. N 3. P. 353-362.

[4] Прохоров И.В. // Журнал вычислительной математики и математической физики. 2002. Т. 42. № 10. С. 1542 1555; Prokhorov I.V. // Computational Mathematics and Mathematical Physics. 2002. V. 42. N 10. P. 1482-1494.

[5] Зимняков Д.А., Тучин В.В. // Квантовая электроника. 2002. T. 32. № 10. C. 849-867; Zimnyakov D.A., Tuchin V.V. // Quantum Electronics. 2002. V. 32. N 10. P. 849-867. doi 10.1070/QE2002v032n10ABEH002307

[6] Терещенко С.А., Данилов А.А., Подгаецкий В.М. // Опт. и спектр. 2007. T. 102. № 5. C. 840-845; Tereshchenko S.A., Danilov A.A., Podgaetsky V.M. // Opt. and Spectr. 2007. V. 102. N 5. P. 771-776.

[7] Зайцев К.И., Гавдуш А.А., Лебедев С.П., Карасик В.Е., Юрченко С.О. // Опт. и спектр. 2015. Т. 118. № 4. C. 582-593; Zaytsev K.I., Gavdush A.A., Karasik V.E., Yurchenko S.O., Lebedev S.P. // Opt. and Spectr. 2015. V. 118. N 4. P. $552-562$. 
[8] Проскурин С.Г., Потлов А.Ю., Фролов С.В. // Квантовая электроника. 2015. T. 45. № 6. C. 540-546; Proskurin S.G., Potlov A.Yu., Frolov S.V. // Quantum Electronics. 2015. V. 45. N 6. P. $540-546$.

[9] Исимару А. Распространение и рассеяние волн в случайно-неоднородных средах. М.: Мир, 1981; Ishimaru A. Wave Propagation and Scattering in Random Media. New York: Academic Press, 1978.

[10] Bal G. // Wave Motion. 2005. V. 43. P. 132-157.

[11] Прохоров И.В., Сущенко А.А. // Акустический журнал. 2015. T. 61. № 3. С. 400-408. doi 10.7868/S0320791915020100;

Prokhorov I.V., Sushchenko A.A. // Acoustical Physics. 2015. V. 61. N 3. P. 368-375. doi 10.1134/S1063771015020104

[12] Прохоров И.В. // Известия РАН. Серия математическая. 2003. Т. 67. № 6. С. 169-192; Prokhorov I.V. // Izvestiya: Mathematics. 2003. V. 67. N 6. P. 1243-1266. doi 10.1070/IM2003v067n06ABEH000463

[13] Прохоров И.В. // Математические заметки. 2009. Т. 86. № 2. C. 256-272; Prokhorov I.V. // Mathematical Notes. 2009. V. 86. N 2. P. 234-248. doi $10.1134 / \mathrm{S} 0001434609070256$

[14] Kovtanyuk A.E., Prokhorov I.V. A boundary-value problem for the polarized-radiation transfer equation with Fresnel interface conditions for a layered medium // Journal of Computational and Applied Mathematics. 2011. V. 235. N 8. P. 2006-2014. doi 10.1016/j.cam.2010.10.001

[15] Прохоров И.В. // Сибирский математический журнал. 2012. T. 53. № 2. C. 377-387; Prokhorov I.V. // Siberian Mathematical Journal. 2012. V. 53. N 2. P. 301-309. doi 10.1134/S0037446612020127

[16] Прохоров И.В. // Журнал вычислительной математики и математической физики. 2013. Т. 53. № 5. С. 753 766. doi 10.7868/S004446691305013X; Prokhorov I.V. // Computational Mathematics and Mathematical Physics. 2013. V. 53. N 5. P. 588-600. doi $10.1134 / \mathrm{S} 0965542513050114$

[17] Amosov A.A. // Journal of Mathematical Sciences. 2013. V. 191. N 2. P. 101-149. doi 10.1007/s10958-013-1308-7

[18] Прохоров И.В., Сущенко А.А. // Сибирский математический журнал. 2015. V. 56. № 4. Р. 922 933. doi 10.17377/smzh.2015.56.415; Prokhorov I.V., Sushchenko A.A. // Siberian Mathematical Journal. 2015. V. 56. N 4. P. 736-745. doi 10.1134/S0037446615040151

[19] Amosov A. Shumarov M. // Applicable Analysis. 2016. V. 95. N 7. P. $1581-1597$. doi 10.1080/00036811.2015.1112000

[20] Prokhorov I.V., Yarovenko I.P., Krasnikova T.V. // Journal of Inverse and Ill-Posed Problems. 2005. V. 13. N 4. P. 365-382. doi 10.1163/156939405775201664

[21] Прохоров И.В., Яровенко И.П. // Оптика и спектроскопия. 2006. T. 101. № 5. С. 817-824; Prokhorov I.V., Yarovenko I.P. Optics and Spectroscopy. 2006. V. 101. N 5. P. 769-776. doi 10.1134/S0030400X0611018X

[22] Prokhorov I.V., Yarovenko I.P., Nazarov V.G. // Inverse Problems. 2008. V. 24. N 2. P. 025019 (13 pp). doi 10.1088/0266$5611 / 24 / 2 / 025019$

[23] Прохоров И.В., Яровенко И.П. // Квантовая электроника. 2010. T. 40. № 1. C. 77-82; Prokhorov I.V., Yarovenko I.P. // Quantum Electronics. 2010. V. 40. N 1. P. 77-82. doi 10.1070/QE2010v040n01ABEH014089

[24] Yarovenko I.P., Prokhorov I.V., Kovtanyuk A.E. // Proceedings of SPIE - The International Society for Optical Engineering. 2016. V. 10035. P. 100350Y (4 pp). doi 10.1117/12.2247989
[25] Крюков П.Г. // Квантовая электроника. 2001. Т. 31. № 2. C. 95-119; Kryukov P.G. // Quantum Electronics. 2001. V. 31. N 2. P. 95-119. doi 10.1070/QE2001v031n02ABEH001906

[26] Иванов А.А., Алфимов М.В., Желтиков А.М. // Успехи физических наук. 2004. Т. 174. № 7. С. 743-763; Ivanov A.A., Alfimov M.V., Zheltikov A.M. // Physics-Uspekhi. 2004. V. 47. N 7. P. 687-704. doi 10.1070/PU2004v047n07ABEH001811

[27] Duck F.A. A physical properties of tissue. A comprehensive reference book. New York: Academic Press, 1990. P. 167-223. 